\title{
PARALLEL SMAC ALGORITHMS TO SOLVE SHALLOW WATER EQUATION WITH UNSTRUCTURED COLLOCATED GRID SYSTEM
}

\author{
Haruka YAMASHITA ${ }^{1} \cdot$ Satoru USHIJIMA ${ }^{2}$ \\ ${ }^{1}$ Student member of JSCE, Graduate Student, Dept. of CERE, Kyoto Univ. (C-cluster, Kyoto 615-8540) \\ ${ }^{2}$ Member of JSCE, Dr. of Eng., Prof. of ACCMS, Kyoto Univ. (Kyoto 606-8501)
}

\begin{abstract}
A computational method to solve shallow water equations has been investigated with an SMAC method which is usually employed in the simulation for incompressible fluids. In particular, this numerical method is implemented in the unstructured collocated grid system with the distributed memory system to increase the parallel efficiency. The developed computational method was applied to the 1D dam-break problem and the free-surface flows in a meandering open channel. As a result of the 1D dam-break simulations, it was confirmed that this method improve the numerical stability. While, in the case of the meandering open channel, it was confirmed that the predicted water depth and depth-averaged velocity distributions are qualitatively in good agreement with the experimental results and that the reasonable parallel efficiencies are attained by parallel computations.
\end{abstract}

KeyWords : shallow water equation, SMAC, MACS, unstructured collocated grid system, parallel computation

\section{INTRODUCTION}

Shallow water equations are widely used in the various hydraulic problems due to its computational efficiency for free-surface flows. The various computational methods have been proposed so far to solve the shallow water equations. As the example of explicit computational methods, MacCormack $\operatorname{method}^{1)}$, multi-level explicit $\operatorname{method}^{2)}$ and many other methods were investigated. On the other hand, there have been some attempts to apply numerical algorithms for incompressible fluids, such as an SMAC method, to solve the shallow water equation system: application of CIP method ${ }^{3)}$, and higher-oder method MACS (MAC method on Collocated grid for Shallow water equations) ${ }^{4), 5)}$, and so on.

Regarding the grid system to solve shallow water equations, structured and unstructured grids are utilized in addition to the curvilinear coordinates. It has been confirmed that MACS is an accurate and stable computational method ${ }^{4), 5)}$ and it was implemented in the curvilinear coordinate system ${ }^{6}$. How- $^{-}$ ever, it is not yet used in the unstructured grid system. Thus, in the present paper, the computational method based on MACS is newly proposed in collocated unstructured grid system. In addition, on the basis of a domain decomposition method, the unstructured cells are decomposed into multiple sub-domains and their computations are simultaneously performed with distributed-memory system. The proposed method was applied to the 1D dam-break problem and open channel flows in a meandering flume, and its validity was examined. The parallel efficiency due to the parallel computations in the case of the meandering open channel is also discussed.

\section{NUMERICAL PROCEDURES}

\section{(1) Governing equations}

By integrating the Reynolds equation in vertical direction, with some assumptions like the hydrostatic distribution, we have two-dimensional continuity equation and momentum equations.

We consider the computational method for the integral form of the shallow water equations in the horizontal fluid area $\Omega$. The continuity equation is given by

$$
\int_{\Omega} \frac{\partial h}{\partial t} d \Omega+\oint_{\partial \Omega} \boldsymbol{M} \cdot \boldsymbol{n} d l=0
$$

where $\partial \Omega$ is the boundary of $\Omega$ and $\boldsymbol{n}$ is a unit normal vector on $\partial \Omega$ which towards outside of the region. As shown in Fig.1, $h$ is the depth of water defined by 


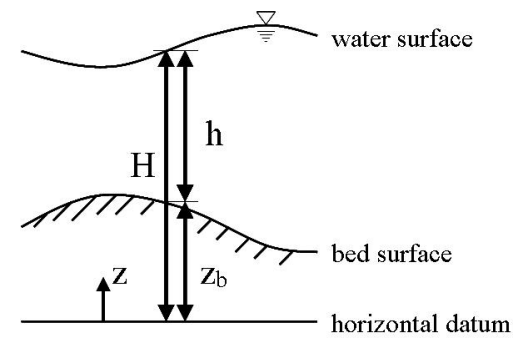

Fig. 1 Water level $H$ from horizontal datum and water depth $h$

$H-z_{b}$, where $H$ and $z_{b}$ are water surface and bottom boundary levels measured from a horizontal datum. $M$ is the vector representing a flow rate of unit width, which given by

$$
M=h \boldsymbol{u},
$$

where $\boldsymbol{u}$ is a depth-averaged velocity vector.

The components of $\boldsymbol{M}$ are $\left(M_{1}, M_{2}\right)$. The momentum equation for $M_{i}$ can be written as

$$
\int_{\Omega}\left(\frac{\partial M_{i}}{\partial t}-\frac{\tau_{i}}{\rho}\right) d \Omega=\oint_{\partial \Omega} \boldsymbol{F}_{i} \cdot \boldsymbol{n} d l
$$

with

$$
\boldsymbol{F}_{i}=-M_{i} \boldsymbol{u}-g \bar{h}\left(H \boldsymbol{k}_{i}\right)+\nu_{M} \nabla M_{i},
$$

where $g$ and $\bar{h}$ are respectively gravity acceleration and a representative water depth that is constant in $\Omega$. Hereafter, the water depth included in the second term on the right hand side of Eq.(4) always means such representative value and $\bar{h}$ is simply represented as $h$.

While the vertically integrated Reynolds stress terms are difficult to deal with, they are approximated as the third term in Eq.(4). The density of the fluid and the diffusion coefficient are $\rho$ and $\nu_{M}$ respectively, which are assumed to be constant. The bottom shear stress is modeled with the usual Manning formula:

$$
\frac{\tau_{i}}{\rho}=-\frac{g n^{2}|\boldsymbol{u}| M_{i}}{h^{4 / 3}} \equiv-\lambda M_{i}
$$

where $n$ is a Manning roughness coefficient and $|\boldsymbol{u}|=$ $\sqrt{u_{1}^{2}+u_{2}^{2}}$.

The Eqs.(1) and (3) are discretized with a finite volume method on unstructured collocated meshes in the following sections.

\section{(2) Unstructured collocated grid}

In the collocated grid system for shallow water equations, the variables $\boldsymbol{M}$ and $h$ are defined in the inner

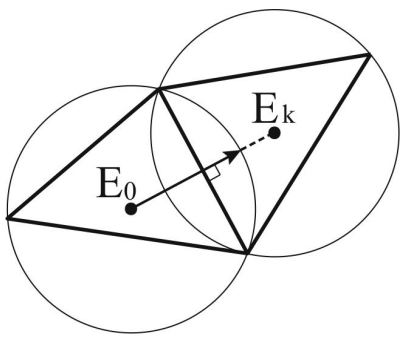

Fig. 2 Neighboring cells shared with same side and circumcenters $E_{0}$ and $E_{k}$

point of a triangular element. This inner point is basically defined as the center of the circumscribed circle, while it is replaced with the center point when the element shape is an obtuse triangle. For convenience, the element inner point is commonly called "cell-center point" hereafter. When the cell-center point is the center of the circumscribed circle, as shown in Fig.2, the side $b_{k}$ of triangular element $E_{0}$ (between element $E_{0}$ and $E_{k}$ ) corresponds to a chord and the straight line between two center points of element $E_{0}$ and $E_{k}$ is normal to the side $b_{k}$. In this case, suppose $\phi$ represents any variable, $\nabla \phi \cdot \boldsymbol{n}$ on the side $b_{k}$ can be approximated by the following relationship:

$$
\left.\nabla \phi \cdot \boldsymbol{n}\right|_{b_{k}} \approx \frac{\phi_{k}-\phi_{0}}{d_{o k}}
$$

where $d_{o k}$ is the distance between two center points.

As done in the computational methods for incompressible fluids using collocated grid system ${ }^{7}$ ), the unknown variables $M$ and $h$ are defined at the cell-center point of each element.

\section{(3) C-ISMAC method for momentum equa- tion}

Similarly to the C-ISMAC method for incompressible fluids ${ }^{8)}$, the momentum equation Eq.(3) is discretized as follows:

$$
\begin{aligned}
& \frac{\hat{M}_{i}^{*}-M_{i}^{n}}{\Delta t} \Delta S= \\
& -\alpha \oint_{\partial \Omega} \hat{M}_{i}^{*} \boldsymbol{u}^{n} \cdot \boldsymbol{n} d l-(1-\alpha) \oint_{\partial \Omega} M_{i}^{n} \boldsymbol{u}^{n} \cdot \boldsymbol{n} d l \\
& +\beta \nu_{M} \oint_{\partial \Omega} \nabla \hat{M}_{i}^{*} \cdot \boldsymbol{n} d l+(1-\beta) \nu_{M} \oint_{\partial \Omega} \nabla M_{i}^{n} \cdot \boldsymbol{n} d l \\
& -g h^{n} \oint_{\partial \Omega}\left(H^{n} \boldsymbol{k}_{i}\right) \cdot \boldsymbol{n} d l-\int_{\Omega} \lambda M_{i}^{n} d \Omega,
\end{aligned}
$$

where $0 \leq \alpha, \beta \leq 1$ and these parameters control the weight of implicit and explicit terms for convection and diffusion. 
In the C-ISMAC method, the following relationship is substituted into Eq.(7):

$$
\hat{M}_{i}^{*}=M_{i}^{n}+\tilde{M}_{i} n
$$

As a result, with the approximation of the integral terms, we obtain the following relationship for the considering element $E_{0}$ :

$$
\begin{aligned}
\tilde{M}_{i 0} & +\frac{\Delta t}{\Delta S} \sum_{k=1}^{3}\left(\alpha \tilde{C}_{i k}-\beta \tilde{D}_{i k}\right) l_{k} \\
& =\frac{\Delta t}{\Delta S} \sum_{k=1}^{3}\left(-C_{i k}+D_{i k}\right) l_{k} \\
& -g h_{0}^{n} \frac{\Delta t}{\Delta S} \sum_{k=1}^{3} H_{0 k}^{n} n_{k i} l_{k}-\lambda M_{i 0}^{n} \Delta t
\end{aligned}
$$

The flux $C_{i k}$ of the convection term in Eq.(9) on side $k$ is defined as

$$
C_{i k}=\left.M_{i}^{n} \boldsymbol{u}^{n}\right|_{k} \cdot \boldsymbol{n}_{k}
$$

With the first-order upwind method, with respect to element $E_{0}$ shown in Fig.2, $C_{i k}$ is estimated as

$$
C_{i k}=\frac{1}{2}\left(U_{k}^{n}-\left|U_{k}^{n}\right|\right) M_{i k}+\frac{1}{2}\left(U_{k}^{n}+\left|U_{k}^{n}\right|\right) M_{i 0},
$$

where $U_{k}^{n}$ stands for $\left.\boldsymbol{u}^{n}\right|_{k} \cdot \boldsymbol{n}_{k} \cdot M_{i k}$ and $M_{i 0}$ are defined at the cell-center of the element $E_{k}$ and $E_{0}$ respectively. On the other hand, with the approximation of Eq.(6), the flux $D_{i k}$ of the diffusion term in Eq.(9) is simply calculated by

$$
\begin{aligned}
D_{i k} & \left.\equiv \nu_{M} \nabla M_{i}^{n}\right|_{k} \cdot \boldsymbol{n}_{k} \\
& \approx \nu_{M} \frac{M_{i k}-M_{i 0}}{d_{o k}}
\end{aligned}
$$

Similarly $\tilde{C}_{i k}$ and $\tilde{D}_{i k}$ mean the fluxes using $\tilde{M}_{i 0}$ and $\tilde{M}_{i k}$. Thus, Eq.(9) forms the linear system of $\tilde{M}_{i 0}$ and $\tilde{M}_{i k}$. The water level $H_{0 k}^{n}$ stands for the $H^{n}$ estimated on the side $k$ of the element $E_{0}$. By solving linear system of Eq.(9), $\tilde{M}_{i 0}$ are obtained for all elements and $\hat{M}_{i 0}^{*}$ are determined by Eq.(8).

In the collocated grid system, it is necessary to remove water-level gradient term and bottom-friction term from the derived $\hat{M}_{i 0}^{*}$ before they are spatially interpolated on the element boundaries. This procedure is necessary to improve the numerical stability and accuracy ${ }^{4) 5)}$. Thus, the tentative $M_{i 0}^{*}$ at cellcenter point is finally calculated as

$$
\begin{aligned}
M_{i 0}^{*} & =M_{i 0}^{n}+\tilde{M}_{i 0} \\
& +g h_{0}^{n} \frac{\Delta t}{\Delta S} \sum_{k=1}^{3} H_{0 k}^{n} n_{k i} l_{k}+\lambda M_{i 0}^{n} \Delta t
\end{aligned}
$$

The derived $M_{i 0}^{*}$ in Eq.(13) is spatially interpolated on element boundaries.

$$
m_{i k}^{*}=f\left(M_{i 0}^{*}, M_{i k}^{*}\right)
$$

In the present method, the interpolation $f$ is performed with a simple arithmetic average of the cellcenter values. With the interpolated value $m_{i k}^{*}$ on a side $b_{k}$ of an element, $m_{i k}^{n+1}$ is obtained by considering the water-level gradient and bottom-friction terms estimated on the element boundary.

$$
\begin{aligned}
& m_{i k}^{n+1}=m_{i k}^{*} \\
& -g h_{b k}^{n} \frac{\Delta t}{\Delta S_{0 k}}\left(H_{k}^{n}-H_{0}^{n}\right) n_{k i} l_{k}-\lambda m_{i k}^{n} \Delta t
\end{aligned}
$$

$h^{n+1}$ is calculated with Eq.(16).

$$
h^{n+1}=h^{n}-\frac{\Delta t}{\Delta S} \sum_{k=1}^{3} \boldsymbol{m}_{k}^{n+1} \cdot \boldsymbol{n}_{k} l_{k},
$$

where $\boldsymbol{m}_{k}^{n+1}=\left(m_{1 k}^{n+1}, m_{2 k}^{n+1}\right)$, which are determined with Eq.(15).

Finally, $\boldsymbol{M}^{n+1}$ at the cell-center is obtained by adding water-level gradient and bottom friction terms to $\boldsymbol{M}^{*}$, which has already been calculated with Eq.(13):

$$
\begin{aligned}
M_{i}^{n+1} & =\frac{1}{1+\lambda \Delta t}\left[M_{i}^{*}\right. \\
& \left.-\frac{\Delta t}{\Delta S} g h^{n+1} \sum_{k=1}^{3} H_{k}^{n} n_{k i} l_{k}\right]
\end{aligned}
$$

\section{(4) SMAC method for shallow water equation}

In the SMAC method for shallow water equations, the water level $H$ is treated implicitly. Thus, instead of Eq.(15), we consider the following relationship:

$$
\begin{aligned}
& m_{i k}^{n+1}=m_{i k}^{*} \\
& -g h_{b k}^{n} \frac{\Delta t}{\Delta S_{0 k}}\left(H_{k}^{n+1}-H_{0}^{n+1}\right) n_{k i} l_{k}-\lambda m_{i k}^{n} \Delta t
\end{aligned}
$$

On the other hand, the tentative cell-boundary value $\hat{m}_{i k}^{*}$ corresponds to $m_{i k}^{n+1}$ in Eq.(15). Thus,

$$
\begin{aligned}
& \hat{m}_{i k}^{*}=m_{i k}^{*} \\
& -g h_{b k}^{n} \frac{\Delta t}{\Delta S_{0 k}}\left(H_{k}^{n}-H_{0}^{n}\right) n_{k i} l_{k}-\lambda m_{i k}^{n} \Delta t
\end{aligned}
$$

Subtracting Eq.(19) from Eq.(18), we obtain

$$
m_{i k}^{n+1}=\hat{m}_{i k}^{*}-g h_{b k}^{n} \frac{\Delta t}{\Delta S_{0 k}}\left(\phi_{k}-\phi_{0}\right) n_{k i} l_{k},
$$

where $\phi_{k}=H_{k}^{n+1}-H_{k}^{n}$. Since the bottom surface level is not changed, $z_{k}^{n+1}-z_{k}^{n}=0$ and $\phi_{k}=h_{k}^{n+1}-$ 
$h_{k}^{n}$. Substituting Eq.(20) into continuity equitation Eq.(16), we obtain the linear relationship for $\phi_{0}$ and $\phi_{k}$ :

$$
\phi_{0}+\sum_{k=1}^{3} \gamma_{k} \phi_{k}=P_{0},
$$

where $\gamma_{k}$ and $P_{0}$ are known values, which are determined from the geometric conditions of unstructured meshes, the divergence of $\hat{\boldsymbol{m}}^{*}$, water depth $h_{b}$ and other constant values. By solving the linear system given by Eq.(21), $h_{0}^{n+1}$ can be determined by

$$
h_{0}^{n+1}=h_{0}^{n}+\phi_{0}
$$

Otherwise, once $m_{i k}^{n+1}$ is calculated from Eq.(20) with the obtained $\phi$ and then we can use Eq.(16) to determine $h^{n+1}$. The latter method allows us to have the better results in terms of the fluid preservation.

\section{(5) Parallelization of numerical procedures}

The computational method was parallelized in a distributed-memory system with a domain decomposition method. The domain decomposition of the generated unstructured cells is performed with METIS ${ }^{9}$, which is a free software for domain decomposition. This method allows us to decrease computational time as well as the necessary memory capacity in each processor.

To solve the simultaneous linear equations due to the implicit methods, a simple SOR method is utilized in this study given by the following iterative equations.

$$
\begin{aligned}
& \phi_{i}^{(k+1)}=\phi_{i}^{(k)}+\omega\left[-\phi_{i}^{(k)}\right. \\
& \left.+\frac{1}{a_{i, i}}\left(b_{i}-\sum_{j \in \boldsymbol{I}(i), j<i} a_{i, j} \phi_{j}^{(k+1)}-\sum_{j \in \boldsymbol{I}(i), j>i} a_{i, j} \phi_{j}^{(k)}\right)\right],
\end{aligned}
$$

where $\phi^{(k)}$ is a scalar at $k$ step computation, $\omega$ is an acceleration parameter and $I(i)$ is the group of the cells neighboring the target element $i$.

When a computation is converged in a target subdomain, the boundary information is communicated by using MPI (Message Passing Interface) ${ }^{10}$ ) which is a library for communicating messages with another processor. Until computations in all sub-domains are converged, the procedure iterates.

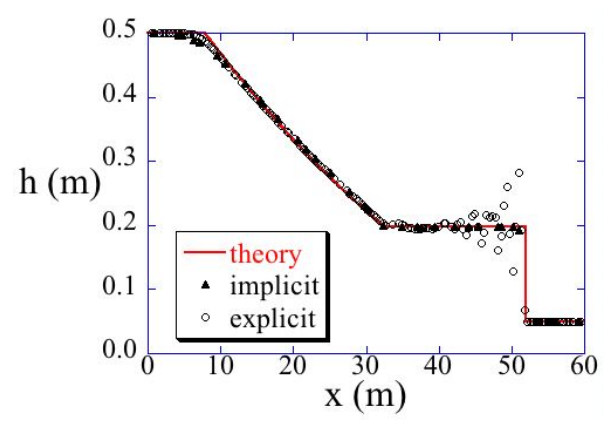

Fig. 3 Water depth at $t=10(\mathrm{~s})$

\section{APPLICABILITY OF COMPUTA- TIONAL METHOD}

\section{(1) 1D dam-break problem}

In order to confirm the numerical stability of the present computational method, it was applied to the 1D dam-break problem. The conditions are as follows: the length in a flowing $x$ direction is $60(\mathrm{~m})$, while initial water depths are $h=0.5(\mathrm{~m})$ at $x \leq 30(\mathrm{~m})$ and $h=0.05(\mathrm{~m})$ at $x>30(\mathrm{~m})$.

Fig.3 shows the water depth at $t=10(\mathrm{~s})$ after the start of dam-breaking. In this figure, "theory" means the theoretical solution, "explicit" means the solution given by explicit method. The result "implicit" stands for the solution given by present method, in which CISMAC method is used to implicitly calculate the tentative $\hat{m}_{i k}^{*}$. In both computational cases of "explicit" and "implicit", the time increment of the computation is $\Delta t=1.0 \times 10^{-1}(\mathrm{~s})$ and the number of elements is 1,040. In Fig.3, the computational results by the explicit method oscillate largely near the uncontentious wave region. On the other hand, the results by the method proposed in this paper are good in agreement with the theoretical solution without such oscillations.

\section{(2) Open channel flows in meandering flume}

\section{a) Experimental conditions}

The measurements of free-surface flows in a meandering flume were performed ${ }^{11)}$. In this experiment, the distributions of velocity and water depth were observed. The experimental flume has ten $2 / \pi$ consecutive unit-bends and straight channels between the bends. Fig.4 shows the definition sketch of one bend.

Table 1 shows the experimental conditions. In all experiments, the gradient of the flume $i$ is $1 / 1000$ along the centerline and the bed is horizontal in the trans- 


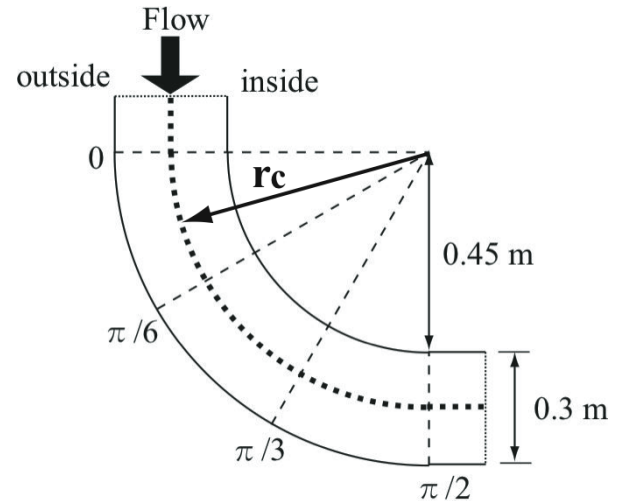

Center line of the experimental flume

Fig. 4 Definition sketch

Table 1 Experimental condition

\begin{tabular}{l|l}
\hline width of the flume $b$ & $0.3(\mathrm{~m})$ \\
centerline radius $r_{c}$ & $0.6(\mathrm{~m})$ \\
length of connection & $0.3(\mathrm{~m})$ \\
material for bed surface & mortar \\
flow rate $Q_{0}$ & $1.96 \times 10^{-3}\left(\mathrm{~m}^{3} / \mathrm{s}\right)$ \\
flow rate per unit width $q_{0}$ & $6.53 \times 10^{-3}\left(\mathrm{~m}^{2} / \mathrm{s}\right)$ \\
mean water depth $h_{m 0}$ & $0.0293(\mathrm{~m})$ \\
\hline
\end{tabular}

verse direction.

The measurements were carried out in the $4 \mathrm{th}, 5 \mathrm{th}$, 6th and 7th bends, in order to observe almost the identical behavior of flows at the same $\theta$ of the bends.

\section{b) Comparisons between experiments and predictions}

In the predictions, initial flow rate per unit width and water depth are same as those in Table 1. On the inlet boundary, flow rate per unit width is kept as $q_{0}$, while the gradient of water depth is 0 along centerline. On the outlet boundary, $h$ is kept as the neutral depth $h_{e}=\left(n^{2} q_{0}^{2} / \sin \theta\right)^{0.3}$ and the gradient of the flow rate is set at 0 . Manning's roughness coefficient of the bed surface $n$ is given by 0.012 . On the side boundaries, non-slip conditions are imposed for velocity and the gradient of $h$ equals 0 .

In the computations, the eddy viscosity $\nu_{t}$ is used instead of $\nu_{m}$ in Eq.(4), in which $\nu_{t}=\alpha U_{*} h$, where $U_{*}$ is friction velocity and $\alpha$ is a constant (0.3). The computations were continued until the flows become almost steady state, in which $t=100$ (s).

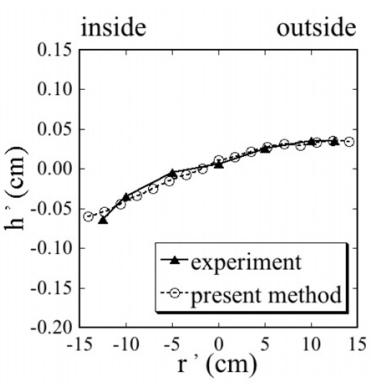

(a) $\theta=0$

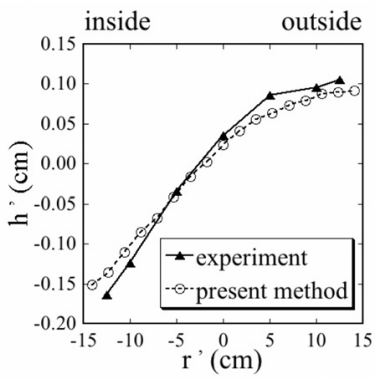

(b) $\theta=\pi / 6$

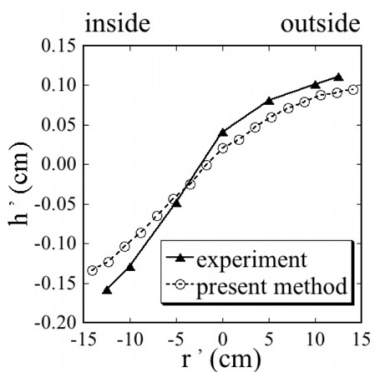

(c) $\theta=\pi / 3$

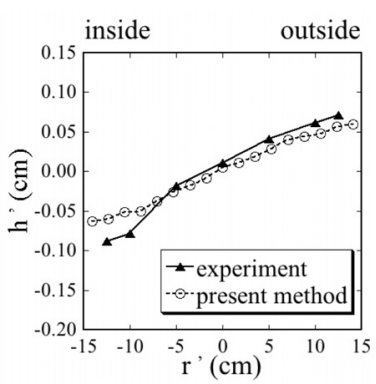

(d) $\theta=\pi / 2$

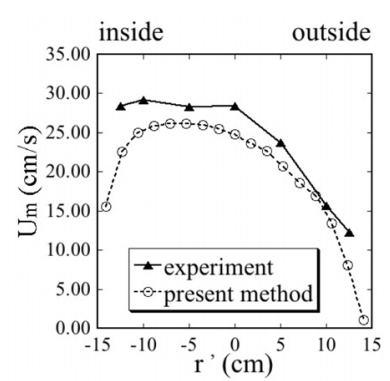

(a) $\theta=0$

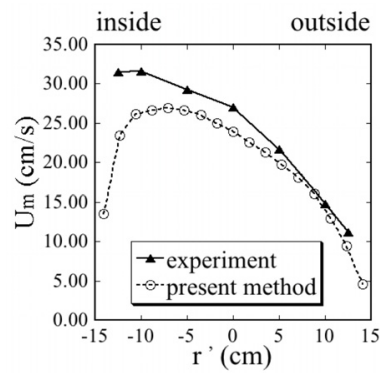

(b) $\theta=\pi / 6$

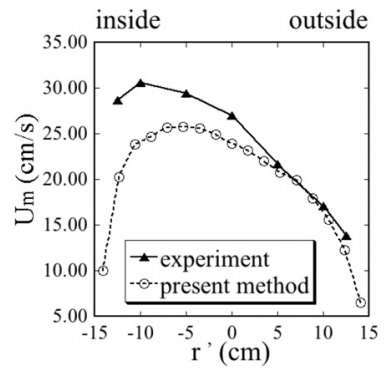

(c) $\theta=\pi / 3$

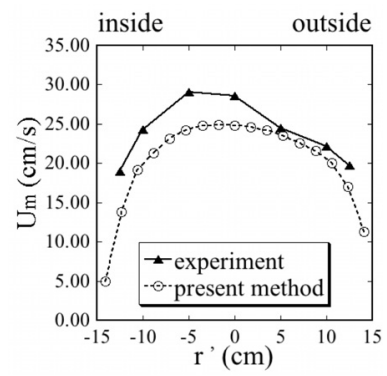

(d) $\theta=\pi / 2$
Fig. 5 Distribution of water-depth and velocity in cross section

Fig.5 shows the distributions of water-depth $h^{\prime}$, where $h-h_{m}$, which is the difference from the averaged water depths $h_{m}$. In these figures, depth-averaged velocity $U_{m}$ in streamwize direction are also presented. In Fig.5, $r^{\prime}$ indicates the transverse distance from the center of the flume. Some discrepancies were found in $h_{m}$, such as $h_{m}=2.93(\mathrm{~cm})$ in experiments and 3.28 $(\mathrm{cm})$ in predictions. As shown in the left column of 


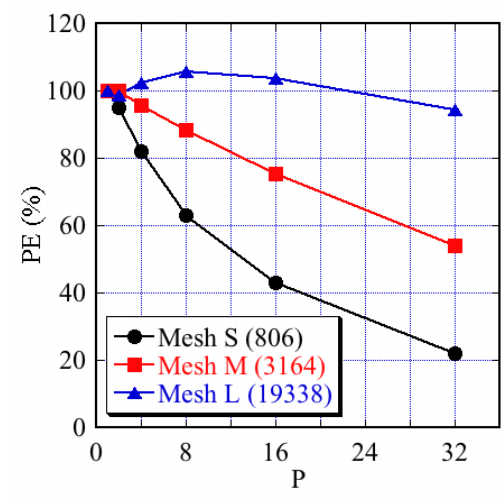

Fig. 6 Relationship between parallel efficiency $P E$ and process number $P$

Fig.5, however, it is shown that the predicted profiles of $h^{\prime}$ are in good agreement with the experimental results. In addition, the distributions of $U_{m}$ are also reasonably predicted as shown in the right column of Fig.5.

The relative error of the flow rate at $\theta$ is represented as $\delta Q_{\theta}=\left(Q_{\theta}-Q_{0}\right) / Q_{0}$, where $Q_{\theta}$ and $Q_{0}$ are flow rate in predictions and experiments, respectively. The relative errors are $\delta Q_{0}=1.29 \times 10^{-2}, \delta Q_{\pi / 6}=0.98 \times$ $10^{-2}, \delta Q_{\pi / 3}=0.87 \times 10^{-2}$ and $\delta Q_{\pi / 2}=1.48 \times 10^{-2}$.

\section{c) Efficiency of parallel computation}

The parallel efficiency was investigated by using different number of unstructured cells for the meandering open channel flows. The computations were performed with the T2K open supercomputer HX600 in Kyoto University. Fig.6 shows the parallel efficiency $P E$ against processor number $P$ using three types of grids: Mesh $S$ (806 cells), Mesh $M$ (3,164 cells) and Mesh $L(19,338$ cells). The parallel efficiency $P E$ is defined by

$$
P E=\frac{T_{1} / T_{p}}{P},
$$

where $T_{k}$ is the computational time using $k$ processors. As shown in this figure, the parallel efficiency of the proposed method increases in large-scale problems, which means the present method is effective for large-scale problems.

\section{CONCLUSION}

In this study, a computational method was proposed to solve shallow water equations in unstructured collocated grid system on the distributed-memory system. The developed computational method was applied to the $1 \mathrm{D}$ dam-break problem to confirm that it improves the numerical stability. In addition, this method was applied to the open channel flows in a meandering flume. As a result, it was shown that the water-depths and depth-averaged velocity distributions are qualitatively in good agreement with the experimental results. In addition, it was demonstrated that the reasonable parallel efficiencies are attained by parallel computations.

\section{REFERENCES}

1) Muneta, N., Shimizu, Y. and Watanabe, Y.: Practical computations of sub-critical and super-critical flows with MacCormack scheme. Civil Engineering Reserch Institute monthly report, No. 448, 1990.

2) Iwasa, Y., Inoue, K. and Mizutori, M.: Hydraulic analysis of overland flood flows by means of numerical method. Annual Journal of Disaster Prevention Research Institute Kyoto University, No. 23B2, pp. 305317,1980

3) Uchida, T. and Kawahara, Y.: Development of a highly accurate numerical method for shallow-water flows with arbitrary boundary shapes. Annual Journal of Hydraulic Engineering, Vol. 50, pp. 799-804, 2006.

4) Ushijima, S., Yamashita, H., Fujioka, S. and Nezu, I.: Prediction method for shallow water equations based on the method for incompressible fluids with collocated grid. Annual Journal of Hydraulic Engineering, Vol. 50, pp. 775-780, 2006.

5) Ushijima, S., Fukutani, A., Yamashita, H. and Nezu, I.: Numerical prediction of sub- and super-critical flows by shallow water equations with macs. Annual Journal of Hydraulic Engineering, Vol. 51, pp. 811-816, 2007.

6) Namihira, A. and Takaki, K.: Application of numerical method for incompressible fluid on collocated grid for depth-averaged flow in generalized coordinate system. Annual Journal of Hydraulic Engineering, Vol. 48, pp. 703-708, 2004.

7) Rhie, C. M. and Chow, W. L.: Numerical study of the turbulent flow past an airfoil with trailing edge separation. AIAA Journal, Vol. 21, pp. 1525-1532, 1983.

8) Ushijima, S. and Nezu, I.: Higher-order implicit method (c-ismac method) for incompressible flows with collocated grid system. Journal of Hydraulic, Coastal and Environmental Engineering, Vol. 719/II-61, pp. 21-30, 2002.

9) Karypis, G. and Kumar, V. Multilevel k-way partitioning scheme for irregular graphs. Journal of Parallel and Distributed Computing, 48(1):96-129, 1998.

10) Pacheco, P. S. Parallel programming with MPI. Morgan Kaufmann Publisher, Inc., 1997.

11) Tamai, N., Ikeuchi, K. and Yamazaki, A.: Experimental analysis of the open channel flow in continuous bends. Journal of Hydraulic, Coastal and Environmental Engineering, Vol. 331, pp. 83-94, 1983.

(Received September 30, 2010) 